\title{
Atypical breast adenosquamous carcinoma following acute myeloid leukemia in a middle-aged woman: A case report
}

\author{
SEYED MEHDI HASHEMI ${ }^{1,2}$, SHOKOUFEH MAHMOUDI SHAN ${ }^{3}$, \\ MAHDI JAHANTIGH $^{4}$ and ABOLGHASEM ALLAHYARI ${ }^{5}$
}

\begin{abstract}
${ }^{1}$ Division of Hematology and Medical Oncology, Department of Internal Medicine, School of Medicine, Zahedan University of Medical Sciences; ${ }^{2}$ Division of Hematology and Medical Oncology, Department of Internal Medicine, Ali-Ebne-Abitaleb Hospital; ${ }^{3}$ Department of Internal Medicine, School of Medicine, Students' Scientific Research Center; ${ }^{4}$ Department of Pathology, School of Medicine, Zahedan University of Medical Sciences, Zahedan; ${ }^{5}$ Division of Hematology and Medical Oncology, Department of Medicine, School of Medicine, Mashhad University of Medical Science, Mashhad, Iran
\end{abstract}

Received July 13, 2016; Accepted October 10, 2016

DOI: $10.3892 / \mathrm{mco} .2017 .1124$

\begin{abstract}
Adenosquamous carcinoma of the breast is a rare cancer that develops as glands and tubules admixed with solid nests of squamous cells in a spindle cell background. Furthermore, its occurrence following AML is also rare. To the best of our knowledge, based on a review of the relevant literature, thus far there have not been any welldocumented cases. In the present case report, we report on a middle-aged woman with a 2 year history of acute myeloid leukemia (AML) who was admitted to hospital due to a mass in the right breast, with concurrent cutaneous lesions on the breast. The clinical and pathological investigations resulted in the diagnosis of adenosquamous carcinoma of the breast. The patient underwent a modified radical mastectomy (MRM). Subsequently, the patient received chemotherapy, involved-field radiation therapy and target therapy. At 9 months after the final cycle of chemotherapy, and while she was on targeted therapy with trastuzumab (6 mg administered every 3 weeks), the patient presented with extensive dermatomal skin lesions. A biopsy report revealed metastatic lesions of invasive ductal carcinoma
\end{abstract}

Correspondence to: Mrs. Shokoufeh Mahmoudi Shan, Department of Internal Medicine, School of Medicine, Students' Scientific Research Center, Zahedan University of Medical Sciences, Khalij Fars Boulevard, Zahedan 43463-98167, Iran

E-mail: sh.mahmoudi_6236@yahoo.com

Abbreviations: AML, acute myeloid leukemia; AUC, area under the curve; MRM, modified radical mastectomy; IFRT, involved-field radiation therapy; HRCT, high-resolution computed tomography; ER, estrogen receptor; PR, progesterone receptor; HER2, human epidermal growth factor receptor type 2 ; HSCT, hematopoietic stem cell transplantation

Key words: acute myeloid leukemia, breast cancer, adenosquamous carcinoma, Iran, metaplastic carcinoma in the abdomen, so chemotherapy resumed with a course lasting for 6 cycles, with the identical treatments, but lacking trastuzumab.

\section{Introduction}

Acute myeloid leukemia (AML) is an aggressive heterogeneous disease that is associated with low survival rates, and the majority of patients who are diagnosed with this malignancy will ultimately die without appropriate treatment (1). It is the most common form of leukemia among adults, and has the lowest rate of survival (2). Furthermore, the incidence of any second malignancy following AML is $2.43 \%$ (3). Second primary breast cancer generally develops in women who are first diagnosed with endometrial colon/rectal, stomach, ovarian, thyroid gland or skin cancers, although its occurrence following AML is rare (4). Additionally, adenosquamous carcinoma of the breast is a rare tumor that exists as one of the subgroups of metaplastic breast carcinoma (5). Metaplastic carcinoma occurs at a prevalence of $1 \%$ of all breast carcinomas (6). In the present study, a case is reported of adenosquamous carcinoma of the breast [invasive ductal carcinoma and squamous cell carcinoma (SCC)] following AML, with metastatic cutaneous lesions of invasive ductal carcinoma on the thorax and abdomen.

\section{Case study}

In May 2010, a 39-year-old Iranian woman was admitted to the Hematology and Medical Oncology Department of the Ali-Ebne-Abitaleb Hospital, Zahedan, Iran, due to non-exertional dyspnea, productive cough and low-grade fever that had lasted over the course of the previous 10 days. On medical evaluation, other symptoms were identified as a decrease in appetite and night sweating. Furthermore, using the routine tests of a peripheral blood smear and complete blood count for pancytopenia, and the modified Westergren method for the erythrocyte sedimentation rate (ESR), pancytopenia and 
an ESR of $122 \mathrm{~mm} / \mathrm{h}$ were observed. Two months prior to admission, the patient had been diagnosed with areas of microcalcifications in her right breast following mammography. The patient's laboratory parameters were as follows: White blood cells, $2.3 \times 10^{3}$; red blood cells, $2.05 \times 10^{6}$ cells $/ \mathrm{ml}$; and platelet count, 66,000 cells $/ \mathrm{ml}$. Based on the pathological report, the patient was diagnosed with AML.

On evaluation in the hematology and medical oncology ward, the patient was diagnosed with AML subtype M4 (acute myelomonocytic leukemia), and this diagnosis also concurred with the results of the bone marrow aspiration, blood smear and flow cytometric analyses. Cytogenetic analysis was not accessible at that time in our center. The patient's chemotherapy was started with a $7+3$ regimen, with a combination of cytarabine and daunorubicin. Subsequently, fever and cough had developed, and the patient coughed up a plug of dense brown mucus. Pathology reports of the plug of dense mucus revealed septate hyphae with regular branches of aspergillosis, necrosis and inflammatory cell infiltration. Furthermore, a follow-up chest computed tomographic (CT) scan revealed blebs in the right lower and middle lobes of the lungs, and high-resolution CT (HRCT) revealed collapse and consolidation in the lateral segment of the right middle lobe, even though the trachea, bronchus and mediastinum were normal. Amphotericin B (50 mg) was administered intravenously (i.v.) for 42 days. After a subsequent 10 days, the patient began to take a regimen and her fever was controlled with imipenem $(750 \mathrm{mg}$ ) and vancomycin $(500 \mathrm{mg})$. At this stage, the treatment continued with itraconazole $(600 \mathrm{mg}$ for 3 days). The patient's treatment was subsequently interrupted for $\sim 2$ years, even though she was a candidate for an allogene bone marrow transplant.

After 2 years, the patient was readmitted to the hospital due to a mass in the right breast, and concurrent skin lesions on the breast (Fig. 1). Following evaluation, the patient was reported to have invasive ductal carcinoma with squamous cell differentiation (SCC) of the breast, with metastasis to the right axillary lymph nodes. Pathology reported metaplastic carcinoma of the breast (adenosquamous carcinoma), and a lymph node involvement (Figs. 2 and 3). Testing for the estrogen receptor (ER) and progesterone receptor $(\mathrm{PR})$ proteins proved to be negative, but human epidermal growth factor receptor type 2 (HER2) was positive. p53, ki-67 and cytokeratin 5/6 also tested positive. Following the diagnosis, the patient underwent a modified radical mastectomy (MRM). During the first week following MRM, the patient took the first course of combination chemotherapy, which comprised a combination of taxotere (160 mg) and carboplatin [6 area under the curve (AUC); $\mathrm{mg} / \mathrm{ml} / \mathrm{min}] /$ trastuzumab $(6 \mathrm{mg} / \mathrm{kg})$ for 6 cycles and, following involved-field radiation therapy (IFRT), targeted therapy was allowed to continue.

The patient presented with extensive dermatomal skin lesions at $\sim 9$ months following the final cycle of chemotherapy, and while she was on targeted therapy with trastuzumab, the patient was followed and her condition was managed as herpes zoster, due to appearance of lesions that induced it. The patient was a candidate for a skin biopsy 14 days afterwards due to the progression of the lesions. During this time, the patient was provided with a regimen of TAC [taxotere $(160 \mathrm{mg})$, adriamycin $(60 \mathrm{mg})$ and cyclophosphamide $(50 \mathrm{mg})]$. The biopsy

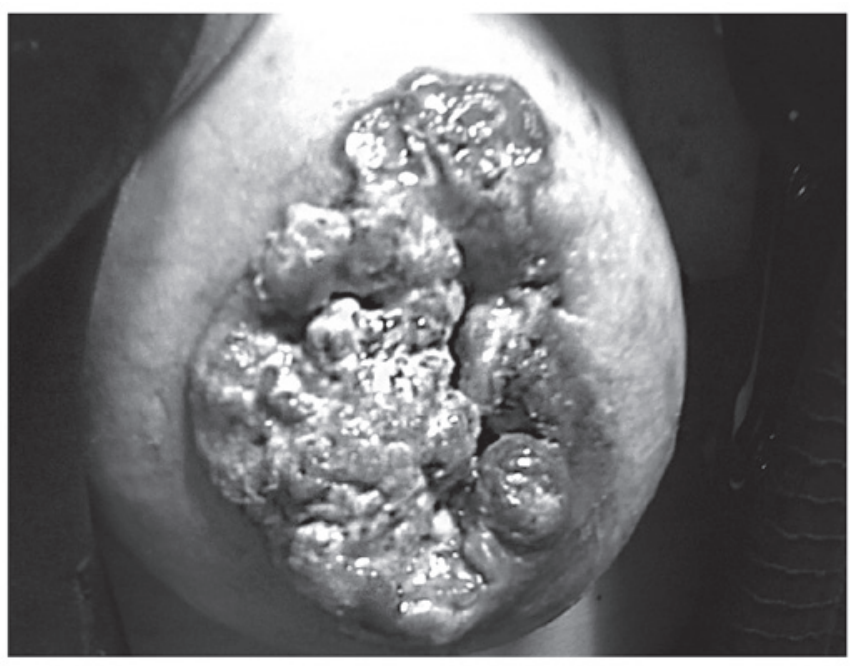

Figure 1. A mass in the right breast of the patient with concurrent skin lesions on her right breast.

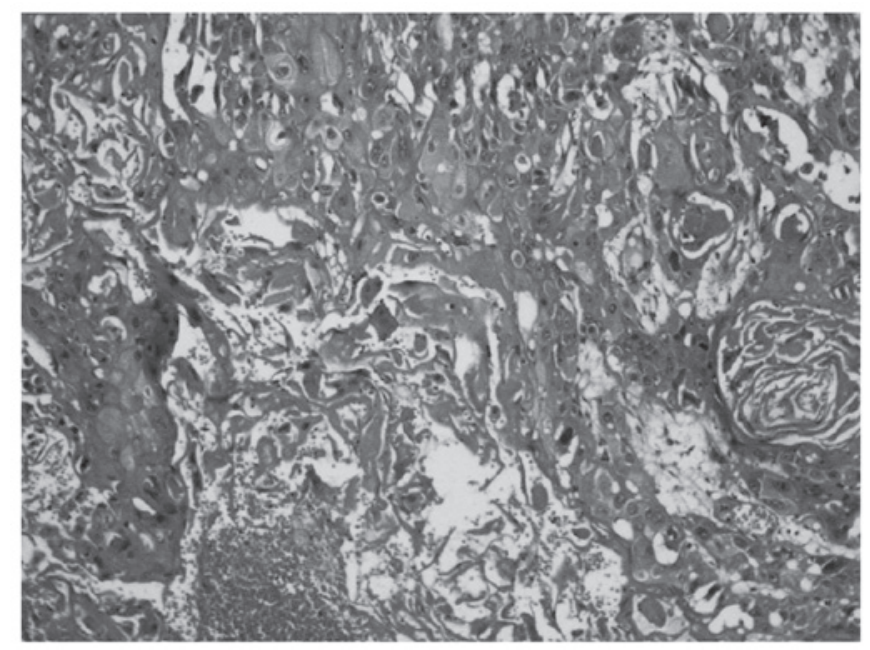

Figure 2. For the breast mass, infiltrative neoplasm consisted of cell plates of squamous cell carcinoma, with focal points of creatine accumulation.

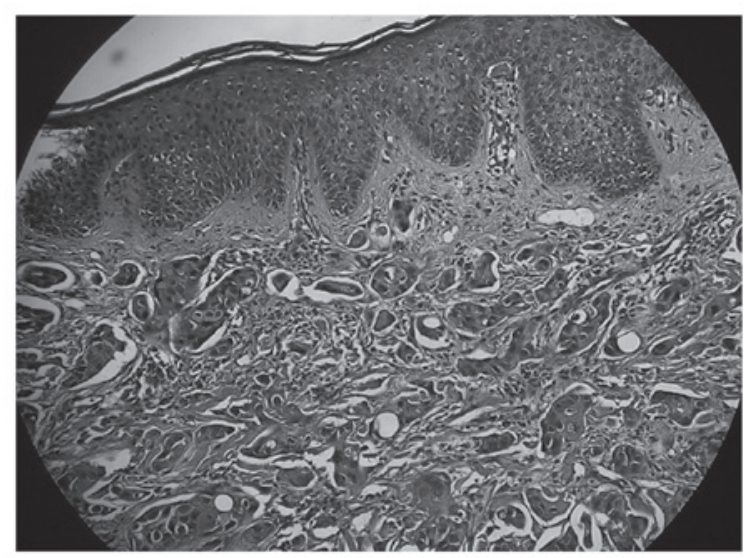

Figure 3. Cutaneous involvement with infiltrative neoplasm, consisting of irregular tubular and glandular structures lined by malignant epithelial cells.

report revealed metastatic lesions of invasive ductal carcinoma to the abdomen, left breast and right axillary region (Fig. 4). 
Table I. Summary of the clinical course and therapeutic measures.

$\begin{array}{lll}\text { Time point } & \text { Clinical event(s) Therapeutic measure(s) }\end{array}$

Last surveillance visit

Day 1 (onset of symptoms)

Second admission

Third admission

(following 2 years' interruption

of the treatment)

Fourth admission

Fifth admission

(9 months following the

final cycle of chemotherapy)

Sixth admission

(14 days later)
Aspergillus infection
Metastatic lesions of
invasive ductal carcinoma
Diagnosis of AML

Mass in the right breast with

concurrent skin lesions on the breast

(adenosquamous carcinoma)

Progression of symptoms

Extensive dermatomal skin lesions

Progression of lesions

(diagnosis of metastatic lesions

of invasive ductal carcinoma)

\begin{abstract}
Liposomal doxorubicin
hydrochloride $(60 \mathrm{mg}$ ) for 6 cycles

$7+3$ regimen, with the combination

of cytarabine and daunorubicin
\end{abstract}

Amphotericin B $(50 \mathrm{mg})$ and the start of the $5+2$ regimen [imipenem $(750 \mathrm{mg})$, vancomycin (500 mg) and itraconazole (600 mg)]

MRM; first course of chemotherapy

[taxotere $(160 \mathrm{mg})$, carboplatin $(50 \mathrm{mg})$

and trastuzumab (6 mg); IFRT

Trastuzumab (6 mg)

TAC regimen [taxotere $(160 \mathrm{mg})$, adriamycin (60 mg) and cyclophosphamide (50 mg)]

Liposomal doxorubicin hydrochloride $(60 \mathrm{mg})$ for 6 cycles
Immunohistochemical (IHC) analysis of the skin lesions revealed a test result of $\mathrm{ER} / \mathrm{PR}^{+++}$; however, HER2 was negative at this time. During this phase, the patient was administered liposomal doxorubicin hydrochloride $(60 \mathrm{mg})$ for 3 weeks. After the completion of 3 cycles, the lesions underwent a marked improvement, and so chemotherapy was continued again for 6 cycles with an identical treatment in all respects, but without trastuzumab during this course of treatment. However, our patient succumbed to mortality due to an endemic hemagglutinin type 1 and neuraminidase type 1 (H1N1) outbreak of influenza, 1 month following the final cycle of the second course of treatment (a summary of the clinical course, and all the therapeutic measures taken, is provided in Table I).

\section{Discussion}

Adenosquamous carcinoma of the breast is a rare cancer that occurs as one of the variants of metaplastic breast carcinoma (5). Adenosquamous carcinoma has a prevalence of $<1 \%$ of all breast cancers (7). It develops as glands and tubules admixed with solid nests of squamous cells in a spindle cell background (8). Second primary breast cancer generally develops in women who are first diagnosed with endometrial, colon/rectal, stomach, ovarian, thyroid gland or skin cancers (4), although its development following AML is rare and, to our best of knowledge, no report of breast cancer following AML has been previously published. However, several cases of second primary AML following breast cancer have been noted in the literature (9-12).

In the present study, our 45-year-old patient had AML. Treatment options included a 7+3 regimen with a combination of cytarabine and daunorubicin, although the patient's treatment was interrupted for $\sim 2$ years. Unusually, our patient

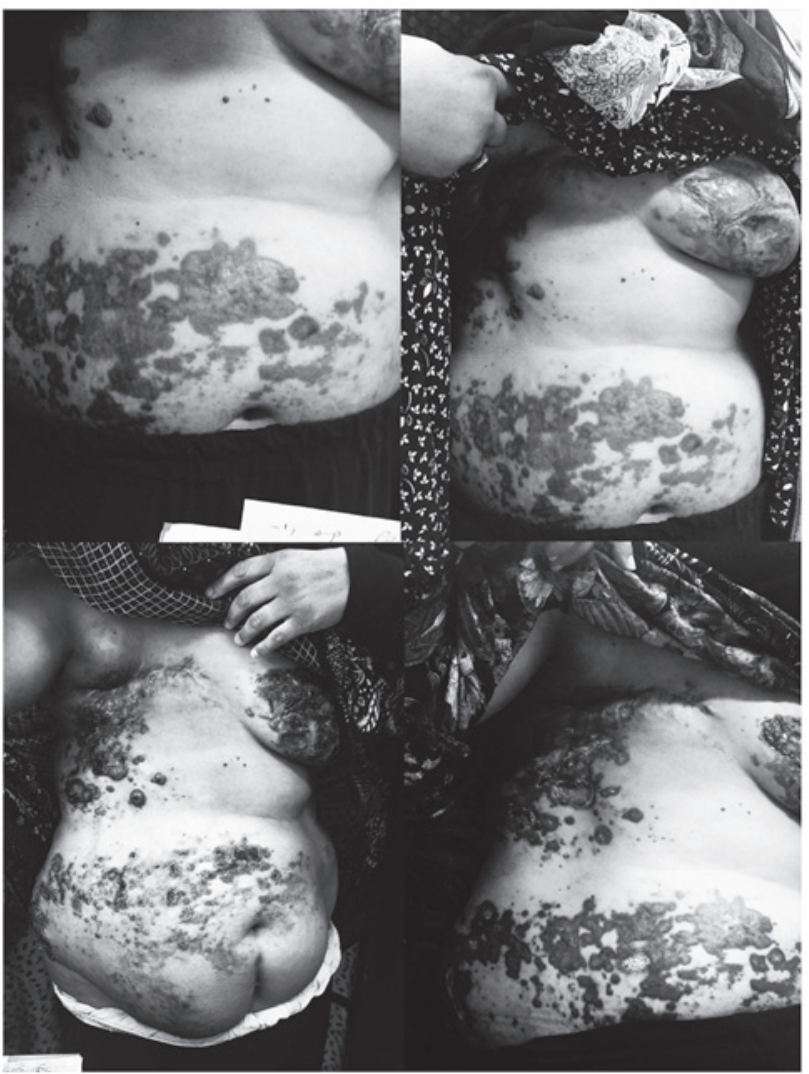

Figure 4. The patient's metastasis skin lesions of invasive ductal carcinoma on the abdomen and left breast and right axillary region. Top panels: Prior to treatment with liposomal doxorubicin hydrochloride; lower panels, following treatment with liposomal doxorubicin hydrochloride.

survived for $\sim 2$ years with only one cycle of induction and without taking any medication, whereas others have reported 
a long-term survival rate for AML of $<10 \%$ (13). Such a survival rate for AML subtype M4 is very unusual, based on the above-mentioned medications and complicated sequels. Several hematological malignancies, including AML, can be effectively treated using allogeneic hematopoietic stem cell transplantation (HSCT) (14). The patient was recommended to have HSCT due to a delay in remission and the M4 subtype of AML, although she refused to continue with the treatment process. Immunocompromised patients, including those who have AML, are at risk from fungal infections, and, although several treatments for fungal infection have been identified, $12-18 \%$ of AML patients still die from fungal infections $(15,16)$. Invasive aspergillosis is the most common fungal infection that can threaten the lives of patients with AML (17). Common antifungal drugs that are used for these patients include amphotericin B, itraconazole, voriconazole, posaconazole and isavuconazole (18). The patient in the present case study was treated with amphotericin B (administered i.v. for 42 days), and subsequently itraconazole (600 mg for 3 days).

The most interesting aspect of the present case report was the feature of primary adenosquamous carcinoma of breast invasive ductal carcinoma and SCC following AML. Adenosquamous carcinoma is one of the types of metaplastic carcinoma that are characterized by the presence of non-epithelial cellular elements (4). Following diagnosis, and due to right axillary lymph nodes metastases, treatment options included MRM and combination chemotherapy [a combination of taxotere $(160 \mathrm{mg})$ and carboplatin (6 AUC; $\mathrm{mg} / \mathrm{ml} / \mathrm{min}) /$ trastuzumab $(6 \mathrm{mg} / \mathrm{kg})]$ for 6 cycles], followed by IFRT, and again, a continuation of the targeted therapy with trastuzumab. According to previous studies, mastectomy is an effective treatment for breast cancer, and it has been demonstrated that a bilateral risk-reducing mastectomy decreases the risk of invasion and metastases in patients with breast cancer by up to $90 \%(19,20)$. Based on a review of the literature, almost $20 \%$ of all breast cancers are HER2-positive, and one of the monoclonal antibodies against the HER2 receptor is trastuzumab, which has been used for HER2-positive breast cancers as an adjuvant treatment and target therapy $(21,22)$. SCC may present with smooth or hyperkeratotic lesions, abscesses, nodules and ulceration of the skin, although nipple discharge is uncommon $(23,24)$, and the symptoms of invasive ductal carcinoma are lumps, itching, burning and pain (25). In the present study, the patient presented with ulceration lesions on the breast with lumps. Breast carcinoma is the most common cancer that causes skin metastases. This metastasis may be presented with nodules on the thoracic wall and abdomen, limbs, head and neck, with ulceration, bleeding and pain $(26,27)$. The patient's specific metastatic invasive ductal carcinoma lesions were observed as ulcerated plaques and nodules.

The patient, who was diagnosed with AML subtype M4, and subsequently with breast cancer (adenosquamous carcinoma) with metastatic lesions of invasive ductal carcinoma, was an unusual case, exhibiting certain differences with respect to other cases that have been described in several other case reports. Our patient had AML, but her treatment was interrupted for $\sim 2$ years. Unexpectedly, the patient was able to survive for $\sim 2$ years with only one cycle of induction, and without taking any medication. Additionally, the most interesting characteristic of the present case was the features of primary adenosquamous carcinoma of breast. In several other cases, certain differences have been demonstrated. According to the study of Geyer et al (28), who discussed five case studies of adenosquamous carcinoma, all the patients were at an age of 54-76 with negative axillary lymph nodes, whereas our patient was 41 years old with positive axillary nodes (28). In addition, in the study of Swathy et al (8), the lesions were identified as being cystic, whereas our patient presented with ulceration lesions on the breast with lumps.

\section{References}

1. Juliusson G, Karlsson K, Lazarevic VL, Wahlin A, Brune M, Antunovic P, Derolf A, Hägglund H, Karbach H, Lehmann S, et al; Swedish Acute Leukemia Registry Group, the Swedish Acute Myeloid Leukemia Group, the Swedish Adult Acute Lymphoblastic Leukemia Group: Hematopoietic stem cell transplantation rates and long-term survival in acute myeloid and lymphoblastic leukemia: Real-world population-based data from the Swedish Acute Leukemia Registry 1997-2006. Cancer 117: 4238-4246, 2011.

2. Robak T and Wierzbowska A: Current and emerging therapies for acute myeloid leukemia. Clin Ther 31: 2349-2370, 2009

3. Ghimire KB and Shah BK: Second primary malignancies in adult acute myeloid leukemia-A US population-based study. Anticancer Res 34: 3855-3859, 2014.

4. Molina-Montes E, Pollán M, Payer T, Molina E, Dávila-Arias C and Sánchez MJ: Risk of second primary cancer among women with breast cancer: A population-based study in Granada (Spain). Gynecol Oncol 130: 340-345, 2013.

5. Agrawal A, Saha S, Ellis IO and Bello AM: Adenosquamous carcinoma of breast in a 19 years old woman: A case report. World J Surg Oncol 8: 44, 2010.

6. Esbah O, Turkoz FP, Turker I, Durnali A, Ekinci AS, Bal O, Sonmez OU, Budakoglu B, Arslan UY and Oksuzoglu B: Metaplastic breast carcinoma: Case series and review of the literature. Asian Pac J Cancer Prev 13: 4645-4649, 2012.

7. Senger JL, Meiers P and Kanthan R: Bilateral synchronous low-grade adenosquamous carcinoma of the breast: A Case report with review of the current literature. Int J Surg Case Rep 14: 53-57, 2015.

8. Swathy PU, Arunalatha P, Chandramouleeswari K, Lily SM and Ramya S: Adenosquamous variant of metaplastic carcinoma of breast - an unusual histological variant. J Clin Diagn Res 9: ED05-ED06, 2015.

9. Renella R, Verkooijen HM, Fioretta G, Vlastos G, Kurtz J, Sappino AP, Schäfer P, Neyroud-Caspar I and Bouchardy C: Increased risk of acute myeloid leukaemia after treatment for breast cancer. Breast 15: 614-619, 2006.

10. Payandeh M, Khodarahmi R, Sadeghi M and Sadeghi E: Appearance of Acute Myelogenous Leukemia (AML) in a Patient with Breast Cancer after Adjuvant Chemotherapy: Case Report and Review of the Literature. Iran J Cancer Prev 8: 125-128, 2015.

11. Noronha V, Berliner N, Ballen KK, Lacy J, Kracher J, Baehring J and Henson JW: Treatment-related myelodysplasia/AML in a patient with a history of breast cancer and an oligodendroglioma treated with temozolomide: Case study and review of the literature. Neuro-oncol 8: 280-283, 2006.

12. Aidan JC, Priddee NR and McAleer JJ: Chemotherapy causes cancer! A case report of therapy related acute myeloid leukaemia in early stage breast cancer. Ulster Med J 82: 97-99, 2013.

13. Yanada $\mathrm{M}$ and Naoe T: Acute myeloid leukemia in older adults. Int J Hematol 96: 186-193, 2012.

14. Berro M, Basquiera AL, Rivas MM, Foncuberta MC, Burgos R, Jaimovich G, Milovic V, Martínez Rolón J, Remaggi G, Alberbide J, et al: Allogeneic hematopoietic stem cell transplantation in the elderly. Predicting the risk for non relapse mortality. Medicina (B Aires) 75: 201-206, 2015.

15. Ducassou S, Rivaud D, Auvrignon A, Vérité C, Bertrand Y, Gandemer V and Leverger G: Invasive Fungal Infections in Pediatric Acute Myelogenous Leukemia. Pediatr Infect Dis J 34: 1262-1264, 2015.

16. Domenech C, Leick-Courtois C, Bienvenu AL, Pracros JP, Picot S, Bleyzac N and Bertrand Y: Improvement in the Outcome of Invasive Aspergillosis in a Pediatric Hematology Department: A 10-Year Review. J Pediatr Hematol Oncol 37: 560-565, 2015. 
17. Gheith S, Ranque S, Bannour W, Ben Youssef Y, Khelif A, Ben Said M, Njah M and Saghrouni F: Hospital environment fungal contamination and aspergillosis risk in acute leukaemia patients in Sousse (Tunisia). Mycoses 58: 337-342, 2015.

18. Karthaus $M$ and Buchheidt D: Invasive aspergillosis: New insights into disease, diagnostic and treatment. Curr Pharm Des 19: 3569-3594, 2013.

19. Morrow M, Winograd JM, Freer PE and Eichhorn JHA: Case records of the Massachusetts General Hospital. Case 8-2013. A 48-year-old woman with carcinoma in situ of the breast. N Engl J Med 368: 1046-1053, 2013.

20. Sismondi P, D'Alonzo M, Pecchio S, Bounous VE, Robba E and Biglia N: Chemoprevention or mastectomy for women at high risk of developing breast cancer. Maturitas 82: 271-273, 2015.

21. Gallagher CM, More K, Masaquel A, Kamath T, Guerin A, Ionescu Ittu R, Nitulescu R, Gauthier Loiselle M, Sicignano N, Butts E, et al: Survival in patients with non metastatic breast cancer treated with adjuvant trastuzumab in clinical practice. Springerplus 5: 395, 2016.

22. Parhi P and Sahoo SK: Trastuzumab guided nanotheranostics: A lipid based multifunctional nanoformulation for targeted drug delivery and imaging in breast cancer therapy. J Colloid Interface Sci 451: 198-211,2015.

23. Gupta C, Malani AK, Weigand RT and Rangineni G: Pure primary squamous cell carcinoma of the breast: A rare presentation and clinicopathologic comparison with usual ductal carcinoma of the breast. Pathol Res Pract 202: 465-469, 2006.
24. Lansbury L, Bath Hextall F, Perkins W, Stanton W and Leonardi Bee J: Interventions for non metastatic squamous cell carcinoma of the skin: systematic review and pooled analysis of observational studies. BMJ347:f6153, 2013.

25. Dye TD, Bogale S, Hobden C, Tilahun Y, Deressa T and Reeler A: Experience of initial symptoms of breast cancer and triggers for action in ethiopia. Int J Breast Cancer 2012: 908547, 2012.

26. Bittencourt MdJS, Carvalho AH, Nascimento BAMd, Freitas LKM and Parijós AMd: Cutaneous metastasis of a breast cancer diagnosed 13 years before. An Bras Dermatol 90: 134-147, 2015.

27. Cabula C, Campana LG, Grilz G, Galuppo S, Bussone R, De Meo L, Bonadies A, Curatolo P, De Laurentiis M, Renne $\mathrm{M}$, et al: Electrochemotherapy in the Treatment of Cutaneous Metastases from Breast Cancer: A Multicenter Cohort Analysis. Ann Surg Oncol 22 (Suppl 3): S442-S450, 2015.

28. Geyer FC, Lambros MB, Natrajan R, Mehta R, Mackay A, Savage K, Parry S, Ashworth A, Badve S and Reis-Filho JS: Genomic and immunohistochemical analysis of adenosquamous carcinoma of the breast. Mod Pathol 23: 951-960, 2010. 